\title{
A POINTWISE CONVERGENCE THEOREM FOR SEQUENCES OF CONTINUOUS FUNCTIONS
}

\author{
BY \\ K. SCHRADER
}

\begin{abstract}
Let $\left\{f_{k}\right\}$ be a sequence of continuous real valued functions defined on an interval $I$ and $N$ a fixed nonnegative integer such that if $f_{k}(x)=f_{i}(x)$ for more than $N$ distinct values of $x \in I$ then $f_{k}(x) \equiv f_{i}(x)$ for $x \in I$. It follows that there is a subsequence $\left\{g_{j}\right\}$ of $\left\{f_{k}\right\}$ such that for each $x$ the subsequence $\left\{g_{j}(x)\right\}$ is eventually monotone. Thus $\lim _{f \rightarrow+\infty} g_{f}(x)=f(x)$ exists for all $x$, where $f$ is an extended real valued function. If $\left|f_{k}(x)\right|$ is bounded for each $x \in I$ then $\lim _{j \rightarrow+\infty} g_{j}(x)=f(x)$ exists as a finite limit for all $x \in I$. For $N=0$ this reduces to picking a monotone subsequence from a sequence of continuous functions whose graphs are pairwise disjoint.
\end{abstract}

1. Introduction. We are interested in sequences $\left\{f_{k}\right\}$ of real valued functions defined on some interval $I$. In particular we are interested in sequences $\left\{f_{k}\right\}$ which satisfy the hypothesis $\mathrm{H}(N)$ or $\mathrm{K}(N)$ for some fixed integer $N$.

$\mathrm{H}(N)$ The sequence $\left\{f_{k}\right\}$ has the property that if $f_{k}(x)=f_{j}(x)$ for more than $N$ distinct values of $x$, then $f_{k}(x) \equiv f_{j}(x)$ for all $x \in I$.

$\mathrm{K}(N)$ The sequence $\left\{f_{k}\right\}$ has the property that if $f_{k}\left(x_{i}\right)=f_{j}\left(x_{i}\right)$ for $x_{1}<x_{2}<\cdots$ $<x_{N+1}$ then $f_{k}(x)=f_{j}(x)$ for $x_{1} \leqq x \leqq x_{N+1}$.

We note that $\mathrm{H}(N)$ makes sense for all nonnegative integers $N$ while $\mathrm{K}(N)$ makes sense for all positive integers $N$. Also, $\mathrm{H}(N)$ implies $\mathrm{K}(N)$ for $N \geqq 1$.

The hypothesis $\mathrm{K}(N)$ will be used in the theorems to follow because this hypothesis on solutions is often encountered in the study of boundary value problems for nonlinear ordinary differential equations of the form

$$
y^{(N+1)}=f\left(x, y, y^{\prime}, \ldots, y^{(N)}\right) \text {. }
$$

If it is known that $f$ is continuous and solutions of initial value problems for (1.1) are unique then $\mathrm{H}(N)$ and $\mathrm{K}(N)$ are equivalent for solutions of (1.1) when $N \geqq 1$. The uniqueness of solutions of initial value problems for (1.1) is not always assumed so it is convenient to use $\mathrm{K}(N)$ rather than $\mathrm{H}(N)$. In this regard, compare the results in [9] and those in [15, Theorem 6.1].

There are a number of papers in which it is assumed that hypothesis $\mathrm{H}(N)$ or $\mathrm{K}(N)$ hold on some family $F$ of functions and in addition it is assumed that there exists some function in $F$ solving any given $N+1$ point boundary value problem. Results following from this type of hypotheses or variations of it plus perhaps

Received by the editors August 8, 1970.

AMS 1969 subject classifications. Primary 3404, 3436, 4020; Secondary 3442.

Key words and phrases. Sequences of functions, pointwise convergence, boundary value problems, uniqueness of solutions.

Copyright (C) 1971, American Mathematical Society 
other assumptions may be found in [2], [3], [6], [10], [11], [12] and [18]. In particular, some uniform convergence theorems are known for a family of functions $F$ satisfying both the "uniqueness" and the "existence" hypotheses for $N+1$ point boundary value problems. See [2, Theorem 2] and [18, Theorem 5] for such results.

Recently there have been a number of papers in ordinary differential equations which have assumed "uniqueness" as in $\mathrm{H}(N)$ or $\mathrm{K}(N)$ or a variation thereof for the family of solutions of (1.1) without assuming the existence of solutions to all $N+1$ point boundary value problems. Results following from such hypotheses plus perhaps other assumptions are found for example in [1], [4], [7], [8], [9], [14], [15], [16], [17], and [19].

In [9], Lasota and Opial show that if $f$ in (1.1) with $N=1$ is continuous on $I \times R^{2}$ for an open interval $I$, if solutions of all initial value problems for (1.1) are unique and exist on $I$ and if $\mathrm{H}(1)$ holds, then all two point boundary value problems have unique solutions. Thus by assuming the "uniqueness" of solutions of all two point boundary value problems for (1.1) with $N=1$ they are able to conclude the "existence" of solutions of all two point boundary value problems for (1.1) with $N=1$. This theorem has been extended to $N=2$ in [5] where it is shown that if $f$ in (1.1) with $N=2$ is continuous on $I \times R^{3}$ for an open interval $I$, if solutions of initial value problems exist on $I$ and if $\mathrm{K}(2)$ holds, then solutions of all two and three point boundary value problems do exist and are unique.

Because of the rather surprising results mentioned above, i.e. "uniqueness" implies "existence" type theorems for solutions of boundary value problems for some ordinary differential equations, one cannot help wondering whether some version of the convergence theorems in [2] and [18] where "uniqueness" and "existence" were both assumed as hypotheses may in fact be true with only the "uniqueness" assumption as hypothesis. Although one cannot conclude the existence of a uniformly convergent subsequence $\left\{g_{j}\right\}$ of an arbitrary bounded sequence of continuous functions $\left\{f_{k}\right\}$ satisfying $\mathrm{K}(N)$ on $I$ as in [2] and [18], we can conclude pointwise convergence for the subsequence $\left\{g_{j}\right\}$. The proofs are constructive in nature and yield a considerable amount of information about the nature of the convergence of the subsequence $\left\{g_{j}\right\}$.

2. Convergence theorems. We begin by stating without proof some known results which we will refer to repeatedly.

THEOREM 2.1. Let I be an interval and $\left\{f_{k}\right\}$ a sequence of continuous real valued functions defined on I having the property that for each $k, j$ either $f_{k}(x) \leqq f_{j}(x)$ holds for all $x \in I$ or else $f_{k}(x) \geqq f_{j}(x)$ holds for all $x \in I$. Then there exists a subsequence $\left\{g_{j}\right\}$ of $\left\{f_{k}\right\}$ which is a monotone sequence on $I$.

COROLLARY 2.2. Let I be an interval and $\left\{f_{k}\right\}$ a sequence of continuous real valued functions defined on I having the property $\mathrm{H}(0)$. Then there exists a subsequence $\left\{g_{j}\right\}$ of $\left\{f_{k}\right\}$ which is a strictly monotone sequence on $I$. 
The next theorem appears as Theorem A in a paper by Ramsey [13] and will be used in later proofs.

THEOREM 2.3. Let $\Gamma$ be an infinite class, and $\mu$ and $r$ positive integers; and let all those subclasses of $\Gamma$ which have exactly $r$ members, or, as we may say, let all $r$ combinations of the members of $\Gamma$ be divided in any manner into $\mu$ mutually exclusive classes $C_{i}(i=1,2, \ldots, \mu)$ so that every r-combination is a member of one and only one $C_{i}$; then, assuming the Axiom of Selections, $\Gamma$ must contain an infinite subclass $\Delta$ such that all the r-combinations of the members of $\Delta$ belong to the same $C_{i}$.

COROLlary 2.4. If $\left\{f_{k}\right\}$ is any sequence of functions defined on an interval I then either there is a subsequence $\left\{h_{j}\right\}$ of $\left\{f_{k}\right\}$ such that if $i \neq j$ then $h_{i}(x) \neq h_{j}(x)$ for any $x \in I$ or else there is a subsequence $\left\{h_{j}\right\}$ of $\left\{f_{k}\right\}$ such that if $i \neq j$ there is some $x \in I$ with $h_{i}(x)=h_{j}(x)$.

Proof. If there are only finitely many distinct functions in $\left\{f_{k}\right\}$ then infinitely many are identically equal and we are done. Thus we assume there are infinitely many distinct functions in $\left\{f_{k}\right\}$ and, by picking a subsequence if necessary, we may assume all the $f_{k}$ are distinct. Let $\mu=r=2$ and $\Gamma=\left\{f_{k}\right\}$. Let $C_{1}=\left\{\left\{f_{k}, f_{j}\right\}: k \neq j\right.$, $f_{k}(x)=f_{j}(x)$ for some $\left.x \in I\right\}$ and $C_{2}=\left\{\left\{f_{k}, f_{j}\right\}: k \neq j, f_{k}(x) \neq f_{j}(x)\right.$ for any $\left.x \in I\right\}$. The result now follows directly from Theorem 2.3.

We are now ready to establish the desired convergence theorems.

THEOREM 2.5. Let I be a bounded interval and $\left\{f_{k}\right\}$ a sequence of continuous real valued functions defined on I. If hypothesis $\mathrm{K}(1)$ is satisfied then there is a subsequence $\left\{g_{j}\right\}$ of $\left\{f_{k}\right\}$ and a sequence of pairwise disjoint intervals $\left\{J_{n}\right\}_{n=0}^{+\infty} \subset I$ such that $I=\bigcup_{n=0}^{+\infty} J_{n} ; J_{0}$ is empty or a single point and $\mu\left(J_{n}\right)=2^{-n} \mu(I)$ for $n=1,2, \ldots$ where $\mu$ is Lebesgue measure; and $\left\{g_{j}\right\}$ is a monotone sequence of functions on each $J_{n}$ for $j$ sufficiently large, depending on $n$.

Proof. Let $J_{1,1}, J_{1,2}$ be disjoint intervals contained in $I$ with $\mu\left(J_{1,1}\right)=\mu\left(J_{1,2}\right)$ $=2^{-1} \mu(I)$ and $J_{1,1} \cup J_{1,2}=I$. Let $\left\{f_{0, k}\right\}=\left\{f_{k}\right\}$. It follows from Corollary 2.4 that either there is a subsequence $\left\{h_{1, j}\right\}$ of $\left\{f_{0, k}\right\}$ such that if $i \neq j$ then $h_{1, i}(x) \neq h_{1, j}(x)$ for any $x$ in $J_{1,1}$ or else there is a subsequence $\left\{h_{1, j}\right\}$ of $\left\{f_{0, k}\right\}$ such that if $i \neq j$ there is some $x \in J_{1,1}$ with $h_{1, i}(x)=h_{1, j}(x)$. In the first case it follows from Corollary 2.2 that there exists a strictly monotone subsequence of $\left\{h_{1, j}\right\}$ on $J_{1,1}$. In the second case it follows from property $\mathrm{K}(1)$ and Theorem 2.1 that there exists a monotone subsequence of $\left\{h_{1, j}\right\}$ on $J_{1,2}$. We denote one of $J_{1,1}, J_{1,2}$ on which there is a monotone sequence by $J_{1}$ and the corresponding subsequence of $\left\{h_{1, j}\right\}$ by $\left\{f_{1, k}\right\}$.

After $J_{n}$ and $\left\{f_{n, k}\right\}$ have been chosen with $\mu\left(J_{n}\right)=2^{-n} \mu(I)$ and $\left\{f_{n, k}\right\}$ a subsequence of $\left\{f_{n-1, k}\right\}$ which is monotone on $J_{n}$ then we consider the interval $I-\bigcup_{k=1}^{n} J_{k}$ and let $J_{n+1,1}, J_{n+1,2}$ be disjoint intervals contained in $I-\bigcup_{k=1}^{n} J_{k}$ with $\mu\left(J_{n+1,1}\right)$ $=\mu\left(J_{n+1,2}\right)=2^{-(n+1)} \mu(I)$ and $J_{n+1,1} \cup J_{n+1,2}=I-\bigcup_{k=1}^{n} J_{k}$. By Corollary 2.4 either there is a subsequence $\left\{h_{n+1, j}\right\}$ of $\left\{f_{n, k}\right\}$ such that if $i \neq j$ then $h_{n+1, i}(x)$ 
$\neq h_{n+1, j}(x)$ for any $x$ in $J_{n+1,1}$ or else there is a subsequence $\left\{h_{n+1, j}\right\}$ of $\left\{f_{n, k}\right\}$ such that if $i \neq j$ there is some $x \in J_{n+1,1}$ with $h_{n+1, i}(x)=h_{n+1, j}(x)$. In the first case it follows from Corollary 2.2 that there exists a strictly monotone subsequence of $\left\{h_{n+1, j}\right\}$ on $J_{n+1,1}$. In the second case it follows from property $\mathrm{K}(1)$ and Theorem 2.1 that there exists a monotone subsequence of $\left\{h_{n+1, j}\right\}$ on $J_{n+1,2}$. We denote one of $J_{n+1,1}, J_{n+1,2}$ on which there is a monotone subsequence by $J_{n+1}$ and the corresponding subsequence of $\left\{h_{n+1, j}\right\}$ by $\left\{f_{n+1, k}\right\}$. By construction we have $\mu\left(J_{n+1}\right)=2^{-(n+1)} \mu(I)$.

Let $x_{0}$ be the unique limit point of the set of midpoints of the intervals $\left\{J_{n}\right\}_{n=1}^{+\infty}$. Here we note that if any of the $J_{n}$ are empty then $I$ was empty or a single point and the proof of the theorem is trivial. Let $\left\{h_{k}\right\}=\left\{f_{k, k}\right\}$ and choose $\left\{g_{j}\right\}$ to be a subsequence of $\left\{h_{k}\right\}$ having the property that $g_{j}\left(x_{0}\right)$ is monotone provided $x_{0} \in I$. If $x_{0} \in J_{n}$ for some $n=1,2, \ldots$ or if $x_{0} \notin I$ we choose $J_{0}=\varnothing$. Otherwise, let $J_{0}=\left\{x_{0}\right\}$. It should now be clear that all the claims in the theorem are satisfied.

To see that it is not possible in general to choose $J_{1}$ with $\mu\left(J_{1}\right)>2^{-1} \mu(I)$, consider the example $f_{k}(x)=k(x-1)$ on $0 \leqq x<2$. To see that it is not possible in general to eliminate the set $J_{0}=\left\{x_{0}\right\}$, consider the example $f_{k}(x)=k^{2} x-k^{2}+k$ on $0 \leqq x \leqq 1$. In this example it is necessarily the case that $J_{0}=\{1\}$. These examples may be modified to give similar examples where the $\left\{f_{k}\right\}$ are uniformly bounded.

The next theorem is the main result of the paper. We observe that this theorem yields detailed information about the convergence behavior of the subsequence $\left\{g_{j}\right\}$.

THEOREM 2.6. Let I be any interval and $\left\{f_{k}\right\}$ a sequence of continuous real valued functions defined on I. If hypothesis $\mathrm{K}(N)$ is satisfied for some positive integer $N$ then there is a subsequence $\left\{g_{j}\right\}$ of $\left\{f_{k}\right\}$ and a sequence of pairwise disjoint intervals $\left\{J_{n}\right\}_{n=0}^{+\infty}$ $\subset I$ such that $I=\bigcup_{n=0}^{+\infty} J_{n}$; each of $J_{0}, J_{1}, \ldots, J_{N-1}$ is empty or a single point and $\left\{J_{n}\right\}_{n=N}^{+\infty}$ each has nonempty interior if I has nonempty interior; and $\left\{g_{j}\right\}$ is a monotone sequence of functions on each $J_{n}$ for $j$ sufficiently large, depending on $n$.

Proof. We assume that $I$ has nonempty interior for otherwise the proof is trivial. Only the case where $I$ is bounded will be treated in detail. If $I$ is unbounded then Theorem 2.5 is still correct if the word "bounded" is omitted from the first sentence and the condition $\mu\left(J_{n}\right)=2^{-n} \mu(I)$ is omitted from the conclusion. The proof of this involves no new ideas except that $J_{n, 1}$ and $J_{n, 2}$ may not be bounded. A similar modification in the proof of Theorem 2.6 for bounded intervals $I$ then yields the result for unbounded intervals.

Let $I$ be a bounded interval with nonempty interior. We will prove the theorem by induction on $N$ and we observe that for $N=1$ the theorem follows from Theorem 2.5. We assume the theorem is true for $1 \leqq N \leqq M$ and will show that this implies it is true for $N=M+1$.

Let $J_{1,1}, J_{1,2}$ be disjoint intervals contained in $I$ with $\mu\left(J_{1,1}\right)=\mu\left(J_{1,2}\right)=2^{-1} \mu(I)$ and $J_{1,1} \cup J_{1,2}=I$. Let $\left\{f_{0, k}\right\}=\left\{f_{k}\right\}$. It follows from Theorem 2.3 with $\mu=M+2$, $r=2, \Gamma \subset\left\{f_{0, k}\right\}$ and $C_{1}, C_{2}, \ldots, C_{M+2}$ appropriately chosen classes of pairs of 
functions from $\Gamma$ that either there is a subsequence $\left\{h_{1, j}\right\}$ of $\left\{f_{0, k}\right\}$ such that if $i \neq j$ then $h_{1, i}(x) \neq h_{1, j}(x)$ for any $x$ in $J_{1,1}$ or else there is an integer $s, 1 \leqq s \leqq M$, and a subsequence $\left\{h_{1, j}\right\}$ of $\left\{f_{0, k}\right\}$ such that if $i \neq j$ then $h_{1, i}(x)=h_{1, j}(x)$ for exactly $s$ distinct values of $x$ in $J_{1,1}$ or else there is a subsequence $\left\{h_{1, j}\right\}$ of $\left\{f_{0, k}\right\}$ such that if $i \neq j$ then $h_{1, i}(x)=h_{1, j}(x)$ for at least $M+1$ distinct values of $x$ in $J_{1,1}$.

In the first case it follows from Corollary 2.2 that there exists a strictly monotone subsequence of $\left\{h_{1, j}\right\}$ on $J_{1,1}$.

In the second case it follows that hypothesis $\mathrm{K}(M+1-s)$ is satisfied by the sequence $\left\{h_{1, j}\right\}$ on $J_{1,2}$ so there is a subsequence $\left\{g_{1, j}\right\}$ of $\left\{h_{1, j}\right\}$ and a sequence of pairwise disjoint intervals $\left\{J_{n}\right\}_{n=0}^{+\infty} \subset J_{1,2}$ such that $J_{1,2}=\bigcup_{n=0}^{+\infty} J_{n}$; each of $J_{0}, J_{1}, \ldots, J_{M-s}$ is empty or a single point and $\left\{J_{n}\right\}_{n=M-s+1}^{+\infty}$ each has nonempty interior; and $\left\{g_{1, j}\right\}$ is a monotone sequence of functions on each $J_{n}$ for $j$ sufficiently large, depending on $n$. Now $\left\{g_{1, j}\right\}$ satisfies hypothesis $\mathrm{K}(s)$ on $J_{1,1}$ so there exists a subsequence $\left\{g_{j}\right\}$ of $\left\{g_{1, j}\right\}$ and a sequence of pairwise disjoint intervals $\left\{I_{n}\right\}_{n=0}^{+\infty} \subset J_{1,1}$ such that $J_{1,1}=\bigcup_{n=0}^{+\infty} I_{n}$; each of $I_{0}, \ldots, I_{s-1}$ is empty or a single point and $\left\{I_{n}\right\}_{n=s}^{+\infty}$ each has nonempty interior; and $\left\{g_{j}\right\}$ is a monotone sequence of functions on each $I_{n}$ for $j$ sufficiently large, depending on $n$. This would complete the proof in the second case.

In the third case it follows from Theorem 2.1 that there exists a subsequence of $\left\{h_{1, j}\right\}$ which is a monotone sequence on $J_{1,2}$.

Since we are finished if case 2 occurs we assume case 1 or case 3 occurred and let $J_{M+1}$ be the one of $J_{1,1}, J_{1,2}$ on which there exists a monotone subsequence of $\left\{h_{1, j}\right\}$ which we denote by $\left\{f_{1, k}\right\}$.

After $J_{M+n}$ and $\left\{f_{n, k}\right\}$ have been chosen with $\mu\left(J_{M+n}\right)=2^{-n} \mu(I)$ and $\left\{f_{n, k}\right\}$ a subsequence of $\left\{f_{n-1, k}\right\}$ which is monotone on $J_{M+n}$ then we consider the interval $I-\bigcup_{k=1}^{n} J_{M+k}$ and let $J_{n+1,1}, J_{n+1,2}$ be disjoint intervals contained in $I-\bigcup_{k=1}^{n} J_{M+k}$ with $\mu\left(J_{n+1,1}\right)=\mu\left(J_{n+1,2}\right)=2^{-(n+1)} \mu(I)$ and $J_{n+1,1} \cup J_{n+1,2}=I-\bigcup_{k=1}^{n} J_{M+k}$. It follows from Theorem 2.3 with $\mu=M+2, r=2, \Gamma \subset\left\{f_{n, k}\right\}$ and $C_{1}, C_{2}, \ldots, C_{M+2}$ appropriately chosen classes of pairs of functions from $\Gamma$ that either there is a subsequence $\left\{h_{n+1, j}\right\}$ of $\left\{f_{n, k}\right\}$ such that if $i \neq j$ then $h_{n+1, i}(x) \neq h_{n+1, j}(x)$ for any $x \in J_{n+1,1}$ or else there is an integer $s, 1 \leqq s \leqq M$, and a subsequence $\left\{h_{n+1, j}\right\}$ of $\left\{f_{n, k}\right\}$ such that if $i \neq j$ then $h_{n+1, i}(x)=h_{n+1, j}(x)$ for exactly $s$ distinct values of $x \in J_{n+1,1}$; or else there is a subsequence $\left\{h_{n+1, j}\right\}$ of $\left\{f_{n, k}\right\}$ such that if $i \neq j$ then $h_{n+1, i}(x)=h_{n+1, j}(x)$ for at least $M+1$ distinct values of $x$ in $J_{n+1,1}$.

In the first case, it follows from Corollary 2.2 that there exists a strictly monotone subsequence of $\left\{h_{n+1, j}\right\}$ on $J_{n+1,1}$.

In the second case it follows that hypothesis $\mathrm{K}(M+1-s)$ is satisfied by the sequence $\left\{h_{n+1, j}\right\}$ on $J_{n+1,2}$ so there is a subsequence $\left\{g_{n+1, j}\right\}$ of $\left\{h_{n+1, j}\right\}$ and a sequence of pairwise disjoint intervals $\left\{K_{n}\right\}_{n=0}^{+\infty} \subset J_{n+1,2}$ such that $J_{n+1,2}=\bigcup_{n=0}^{+\infty} K_{n}$; each of $K_{0}, K_{1}, \ldots, K_{M-s}$ is empty or a single point and $\left\{K_{n}\right\}_{n=M-s+1}^{+\infty}$ each has nonempty interior and $\left\{g_{n+1, j}\right\}$ is a monotone sequence of functions on each $K_{n}$ for $j$ sufficiently large, depending on $n$. Now $\left\{g_{n+1, j}\right\}$ satisfies hypothesis $\mathrm{K}(s)$ on 
$J_{n+1,1}$ so there exists a subsequence $\left\{g_{j}\right\}$ of $\left\{g_{n+1, j}\right\}$ and a sequence of pairwise disjoint intervals $\left\{L_{n}\right\}_{n=0}^{+\infty} \subset J_{n+1,1}$ such that $J_{n+1,1}=\bigcup_{n=0}^{+\infty} L_{n}$; each of $L_{0}, L_{1}, \ldots$, $L_{s-1}$ is empty or a single point and $\left\{L_{n}\right\}_{n=s}^{+\infty}$ each has nonempty interior; and $\left\{g_{j}\right\}$ is a monotone sequence of functions on each $L_{n}$ for $j$ sufficiently large, depending on $n$. This would complete the proof in the second case.

In the third case it follows from Theorem 2.1 that there exists a subsequence of $\left\{h_{n+1, j}\right\}$ which is a monotone sequence on $J_{n+1,2}$.

Since we are finished if case 2 occurred we assume case 1 or case 3 occurred and let $J_{M+n+1}$ be the one of $J_{n+1,1}, J_{n+1,2}$ on which there exists a monotone subsequence of $\left\{h_{n+1, j}\right\}$ which we denote by $\left\{f_{n+1, k}\right\}$.

Assuming case 2 never occurs at any stage of the induction then we let $x_{0}$ be the unique limit point of the set of midpoints of the intervals $\left\{J_{M+n}\right\}_{n=1}^{+\infty}$. Let $\left\{h_{k}\right\}=\left\{f_{k, k}\right\}$ and choose $\left\{g_{j}\right\}$ to be a subsequence of $\left\{h_{k}\right\}$ having the property that $\left\{g_{j}\left(x_{0}\right)\right\}$ is monotone provided $x_{0} \in I$. If $x_{0} \in J_{M+n}$ for some $n=1,2, \ldots$ or if $x_{0} \notin I$ we choose $J_{0}=J_{1}=\cdots=J_{M}=\varnothing$; otherwise choose $J_{0}=\left\{x_{0}\right\}, J_{1}=J_{2}=\cdots=J_{M}=\varnothing$. This completes the proof.

COROllary 2.7. Let I be any interval and $\left\{f_{k}\right\}$ a sequence of continuous real valued functions defined on I. If hypothesis $\mathrm{K}(N)$ is satisfied for some positive integer $N$ or if hypothesis $\mathrm{H}(N)$ is satisfied for some nonnegative integer $N$ then there is a subsequence $\left\{g_{j}\right\}$ of $\left\{f_{k}\right\}$ and an extended real valued function $f$ such that $\lim _{j \rightarrow+\infty} g_{j}(x)$ $=f(x)$ for all $x \in I$.

COROLlary 2.8. Let I be any interval and $\left\{f_{k}\right\}$ a sequence of continuous real valued functions defined on I such that $\left\{\left|f_{k}(x)\right|\right\}$ is bounded for each $x \in I$. If hypothesis $\mathrm{K}(N)$ is satisfied for some positive integer $N$ or if hypothesis $\mathrm{H}(N)$ is satisfied for some nonnegative integer $N$ then there is a subsequence $\left\{g_{j}\right\}$ of $\left\{f_{k}\right\}$ and a real valued function $f$ such that $\lim _{j \rightarrow+\infty} g_{j}(x)=f(x)$ for all $x \in I$.

The conclusions of Theorem 2.6, Corollary 2.7 and Corollary 2.8 are valid for any interval $I$ which can be written as a countable union of intervals on each of which the hypothesis of these theorems is satisfied. For example, the generalization of Theorem 2.6 would be

THEOREM 2.9. Let $I$ be any interval such that $I=\bigcup_{m=1}^{+\infty} I_{m}$ where $I_{j} \cap I_{m}=\varnothing$ for $j \neq m$ and let $\left\{f_{k}\right\}$ be a sequence of continuous real valued functions defined on $I$. If hypothesis $\mathrm{K}\left(N_{n}\right)$ is satisfied by $\left\{f_{k}\right\}$ on $I_{n}$ for some positive integer $N_{n}$ then there is a subsequence $\left\{g_{j}\right\}$ of $\left\{f_{k}\right\}$ and a sequence of pairwise disjoint intervals $\left\{J_{n}\right\}_{n=0}^{+\infty} \subset I$ such that $I=\bigcup_{n=0}^{+\infty} J_{n}$ and $\left\{g_{j}\right\}$ is a monotone sequence of functions on each $J_{n}$ for $j$ sufficiently large, depending on $n$.

Proof. The proof is a standard diagonalization argument arrived at by applying Theorem 2.6 to each $I_{n}$ so is not presented. 
It should be pointed out that the sequence of functions $f_{k}(x)=k|x|(1-|x|)^{k}$ on $-\frac{1}{2} \leqq x \leqq \frac{1}{2}$ has property $\mathrm{H}(3)$ and $\lim _{k \rightarrow+\infty} f_{k}(x)=0$ for all $x$ in $-\frac{1}{2} \leqq x \leqq \frac{1}{2}$. However, the convergence of any subsequence is not uniform since $f_{k}(1 /(k+1))$ $=(k /(k+1))^{1+k} \rightarrow e^{-1}$ as $k \rightarrow+\infty$. This shows that Dini's theorem concluding the uniform convergence of any monotone sequence of continuous functions converging pointwise to a continuous function on a compact interval does not generalize to any subsequence of $\left\{f_{k}\right\}$. This presents a contrast with the results in [2, Theorem 2] and [18, Theorem 5] where uniform convergence of a subsequence is obtained by assuming both "uniqueness" and "existence" of solutions of $N+1$ point boundary value problems.

One might wonder whether the method of proof used in [2, Theorem 2] and [18, Theorem 5] might provide an easier method for concluding the pointwise convergence of a subsequence of $\left\{f_{k}\right\}$. In these proofs it was shown, in particular, that if $N+1$ distinct points $x_{1}<x_{2}<\cdots<x_{N+1}$ are chosen in $I$ and $\left\{g_{j}\right\}$ is any subsequence of $\left\{f_{k}\right\}$ such that $\lim _{j \rightarrow+\infty} g_{j}\left(x_{i}\right)=y_{i}$ exists as a finite number for $i=1,2, \ldots, N+1$ then $\left\{g_{j}\right\}$ actually converges at all points in $I$. The following example with $N=1$ satisfies $\mathrm{H}(1)$ and shows that the above-mentioned property is not necessarily true with our hypotheses. In fact, in this example the sequence $\left\{g_{j}\right\}=\left\{f_{k}\right\}$ converges only at the points $x_{1}=0$ and $x_{2}=2$. Let

$$
\begin{array}{rlrl}
f_{k}(x) & =\left(1-k^{-1}\right)(x-1)+1 & & \text { if } 0 \leqq x \leqq 1, \\
& =-\left(1+k^{-1}\right)(x-1)+1 & \text { if } 1<x \leqq 2,
\end{array} \text { for } k \text { odd, }
$$

and

$$
\begin{aligned}
f_{k}(x) & =-\left(1+k^{-1}\right)(x-1)-1 & & \text { if } 0 \leqq x \leqq 1, \\
& =\left(1-k^{-1}\right)(x-1)-1 & & \text { if } 1<x \leqq 2,
\end{aligned}
$$

If $x_{1}=0, x_{2}=2$ and $\left\{g_{j}\right\}=\left\{f_{k}\right\}$ then $\lim _{j \rightarrow+\infty} g_{j}(0)=0$ and $\lim _{j \rightarrow+\infty} g_{j}(2)=0$ but $\left\{g_{j}\right\}$ does not converge at any other points in $0 \leqq x \leqq 2$. There is of course some other subsequence of $\left\{f_{k}\right\}$ which converges at every point by Theorem 2.6. It is not hard to see that for $\left\{h_{j}\right\}$ chosen to be the subsequence of $\left\{f_{k}\right\}$ with odd indices we get convergence to

$$
\begin{aligned}
h(x) & =x & & \text { if } 0 \leqq x \leqq 1, \\
& =2-x & & \text { if } 1<x \leqq 2,
\end{aligned}
$$

and for $\left\{h_{j}\right\}$ chosen to be the subsequence of $\left\{f_{k}\right\}$ with even indices we get convergence to

$$
\begin{aligned}
h(x) & =-x & & \text { if } 0 \leqq x \leqq 1 \\
& =x-2 & & \text { if } 1<x \leqq 2 .
\end{aligned}
$$

We present one last theorem which would appear to give, by repeated application, an alternative method for proving Theorem 2.6. This suggested proof does not seem to work because one may end up with an uncountable Cantor type set of 
Lebesgue measure zero on which the subsequence $\left\{g_{j}\right\}$ is not yet known to converge. One can however prove the existence of a subsequence $\left\{g_{j}\right\}$ of $\left\{f_{k}\right\}$ which converges almost everywhere to an extended real valued function on $I$ in this manner.

THEOREM 2.10. Let I be a bounded interval and $\left\{f_{k}\right\}$ a sequence of continuous real valued functions defined on I. If hypothesis $\mathrm{K}(N)$ is satisfied for some $N \geqq 1$ then there is a subsequence $\left\{g_{j}\right\}$ of $\left\{f_{k}\right\}$ and an interval $J \subset I$ with $\mu(J)=(N+1)^{-1} \mu(I)$ such that $\left\{g_{j}\right\}$ is a monotone sequence on $J$.

Proof. Let $I_{1}, I_{2}, \ldots, I_{N+1}$ be pairwise disjoint intervals contained in $I$ with $\mu\left(I_{k}\right)=(N+1)^{-1} \mu(I)$ for $k=1,2, \ldots, N+1$ and $I=\bigcup_{k=1}^{N+1} I_{k}$. It follows from Theorem 2.3 and property $\mathrm{K}(N)$ that one of the intervals $I_{1}, \ldots, I_{N+1}$ may be chosen to be the interval $J$ on which there is a monotone subsequence $\left\{g_{j}\right\}$ of $\left\{f_{k}\right\}$.

The result in Theorem 2.10 is the best possible in that in general there need not be any interval contained in $I$ having measure greater than $(N+1)^{-1} \mu(I)$ on which any subsequence of $\left\{f_{k}\right\}$ is monotone. This can be seen by examining the functions $f_{k}(x)=k^{-1} \sin x$ with $I=[\pi / 2,(\pi / 2)+N \pi]$.

The theorems in this paper are of course not true in general if hypotheses $\mathrm{K}(N)$ and $\mathrm{H}(N)$ are omitted entirely. The example $f_{k}(x)=\sin k x \pi$ for $0 \leqq x \leqq 1$ has the property that there is no subsequence $\left\{g_{j}\right\}$ which converges almost everywhere to any function $f$. Indeed, for this example, if $\lim _{j \rightarrow+\infty} g_{j}(x)=f(x)$ a.e. then $\lim _{j \rightarrow+\infty} g_{j}(x) f(x)=f^{2}(x)$ a.e., so by the Riemann Lebesgue lemma $\int_{0}^{1} f^{2}(x) d x=0$ and $f=0$ a.e. implying $\lim _{j \rightarrow+\infty} \int_{0}^{1} g_{j}^{2}(x) d x=0$. Direct computation shows that $\int_{0}^{1} f_{k}^{2}(x) d x=\frac{1}{2}$ for all $k=1,2, \ldots$ This gives a contradiction.

The only property of continuous functions which was used in any of the theorems of this paper was the intermediate value property, so that every theorem remains valid if the continuous functions $\left\{f_{k}\right\}$ are replaced for example by any sequence of derivatives $\left\{f_{k}^{\prime}\right\}$.

\section{REFERENCES}

1. P. Bailey and L. Shampine, Existence from uniqueness for two point boundary value problems, J. Math. Anal. Appl. 25 (1969), 569-574. MR 39 \#530.

2. E. F. Beckenbach, Generalized convex functions, Bull. Amer. Math. Soc. 43 (1937), 363-371.

3. P. Hartman, Unrestricted n-parameter families, Rend. Circ. Mat. Palermo (2) 7 (1958), 123-142. MR 21 \#4211.

4. L. Jackson, Subfunctions and second-order ordinary differential inequalities, Advances in Math. 2 (1968), 307-363. MR 37 \#5462.

5. L. Jackson and K. Schrader, Existence and uniqueness of solutions of boundary value problems for third order differential equations, J. Differential Equations 9 (1971), 46-54.

6. - On second order differential inequalities, Proc. Amer. Math. Soc. 17 (1966), 1023-1027. MR 34 \#401.

7. - Subfunctions and third order differential inequalities, J. Differential Equations 8 (1970), 180-194.

8. G. Klaasen, Dependence of solutions on boundary conditions for second order ordinary differential equations, J. Differential Equations 7 (1970), 24-33. 
9. A. Lasota and Z. Opial, On the existence and uniqueness of solutions of a boundary value problem for an ordinary second-order differential equation, Colloq. Math. 18 (1967), 1-5. MR 36 \#2871.

10. R. Mathsen, $\lambda(n)$-parameter families, Canad. Math. Bull. 12 (1969), 185-191. MR 39 \#7191.

11. Z. Opial, On a theorem of O. Arama, J. Differential Equations 3 (1967), 88-91. MR 34 \#6194.

12. M. M. Peixoto, Generalized convex functions and second order differential inequalities, Bull. Amer. Math. Soc. 55 (1949), 563-572. MR 10, 686.

13. F. Ramsey, On a problem of formal logic, Proc. London Math. Soc. (2) 30 (1930), 264-286.

14. K. Schrader, A note on second order differential inequalities, Proc. Amer. Math. Soc. 19 (1968), 1007-1012. MR 38 \#2416.

15. - Existence theorems for second order boundary value problems, J. Differential Equations 5 (1969), 572-584. MR 39 \#532.

16. K. Schrader and P. Waltman, An existence theorem for nonlinear boundary value problems, Proc. Amer. Math. Soc. 21 (1969), 653-656. MR 39 \#533.

17. L. Shampine, Existence and uniqueness for nonlinear boundary value problems, J. Differential Equations 5 (1969), 346-351. MR 39 \#534.

18. L. Tornheim, On n-parameter families of functions and associated convex functions, Trans. Amer. Math. Soc. 69 (1950), 457-467. MR 12, 395.

19. P. Waltman, Existence and uniqueness of solutions to a nonlinear boundary value problem, J. Math. Mech. 18 (1968), 585-586. MR 38 \#3504.

UNIVERSITY OF MisSOURI,

Columbia, Missouri 65201 